\title{
Embedded fuzzy controller for water level control
}

\author{
Javier Eduardo Martínez Baquero ${ }^{1}$, Jairo David Cuero Ortega ${ }^{1}$, Robinson Jiménez-Moreno ${ }^{2}$ \\ ${ }^{1}$ Faculty of Basic Sciences and Engineering, Universidad de los Llanos, Villavicencio, Colombia \\ ${ }^{2}$ Faculty of Engineering, Mechatronic Engineering, Universidad Militar Nueva Granada, Bogota, Colombia
}

\begin{tabular}{|c|c|}
\hline Article Info & ABSTRACT \\
\hline Article history: & This article presents the design of a fuzzy controller embedded in a \\
\hline Received Apr 27, 2021 & system. The fuzzy system's construction is based on a classical proportional \\
\hline Revised Jul 15, 2021 & and derivative controller, where inputs of error and its derivate depend on \\
\hline Accepted Aug 2, 2021 & $\begin{array}{l}\text { the difference between the desired setpoint and the actual level; the goal is to } \\
\text { control the water level of coupled tanks. The process is oriented to control }\end{array}$ \\
\hline Keywords: & $\begin{array}{l}\text { based on the knowledge that facilitates the adjustment of the output variable } \\
\text { without complex mathematical modeling. In different response tests of the }\end{array}$ \\
\hline $\begin{array}{l}\text { Coupled tanks } \\
\text { Embedded svstem }\end{array}$ & $\begin{array}{l}\text { fuzzy controller, a maximum over-impulse greater than } 8 \% \text { or a steady-state } \\
\text { error greater than } 2.1 \% \text { was not evidenced when varying the setpoint. }\end{array}$ \\
\hline
\end{tabular}

This is an open access article under the CC BY-SA license.

Embedded system

Fuzzy control

\begin{abstract} and derivative controller, where inputs of error and its derivate depend on based on the knowledge that facilitates the adjustment of the output variable fuzzy controller, a maximum over-impulse greater than $8 \%$ or a steady-state
\end{abstract}

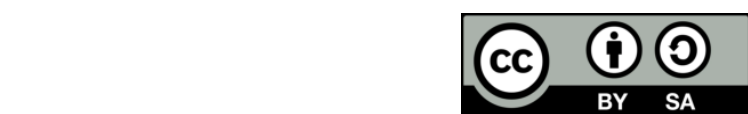

\section{Corresponding Author:}

Javier Eduardo Martinez Baquero

Faculty of Basic Sciences and Engineering, Universidad de los Llanos

Transversal 25 \#13-34, Villavicencio, Colombia

Email: jmartinez@unillanos.edu.co

\section{INTRODUCTION}

In industrial automation processes, the control of variables is required to maintain the desired operating conditions. For example, Abdullah and Ali present in [1] an application of motor's torque control using a classical controller proportional, integral, derivative, or proportional integral derivative (PID) controller. Rachedi et al. in [2] presents a speed controller design for a wind turbine is presented, and in [3] the control of a DC-DC converter. For water management systems oriented to applications such as precision agriculture [4], the use of control strategies is also required, which can be very varied, as explained in [5]-[7].

System modeling can be highly complex and present non-linearities, making controller design difficult. Techniques such as fuzzy systems allow obtaining a very close approximation of their response without an established mathematical model [8], [9]. Since the mathematical model is a necessity for the design of control strategies, fuzzy logic presents multiple examples of its adaptation to avoid this requirement [10]-[12]. The applications range from control of robotic arms [13], control of the speed of motors [14], including the case previously referred to for speed control of a wind turbine [15].

The basic PID control scheme in fuzzy systems is based on Mamdani and Takagi Sugeno models [16], [17], in such a way that by being able to control an actuator [18] such as a water filling solenoid valve to tanks, a fuzzy controller can be applied to this case [19]. Unlike the examples presented previously, lowcost portable systems capable of embedding a fuzzy algorithm for level control are rare; this is precisely the scope of this work. Control algorithms can be embedded at low cost into microcontrollers [20], [21], in the same way that fuzzy systems are found in state-of-the-art, oriented to internet of things (IoT) data acquisition [22], and implemented in microcontrollers [23] as low-cost solutions. To address this last issue, some authors have embedded a fuzzy controller into a microcontroller system that makes measurements of the level and controls an actuator to regulate a storage tank's water level. Those previous works are about intelligent 
algorithms [24], [25]. The paper is divided into four sections. The first is the previous introduction; then, the second section explains the research method, the fuzzy system, and the tank level system. The third section exposes the results and discussion of the level control and its analysis, and finally, section four reveals the conclusions achieved.

\section{RESEARCH METHOD}

The level control system consists of a workbench with two coupled water storage tanks as shown in Figure 1. The water is entered, employing an electric pump activated using a relay commanded by the micro controlled controller. The controller design is based on two stages, the first corresponding to the hardware and the second to the software. These are described next.

\subsection{Hardware}

The MBED NXP LPC1768 microcontroller as shown in Figure 2 was chosen for programming the fuzzy algorithm, reading the ultrasonic sensor for level, and the command of the activation relay of the filling pump. This microcontroller has ARM Cortex-M3 core 32 Bit running at $96 \mathrm{MHz}$, includes $512 \mathrm{~KB}$ flash memory, $32 \mathrm{~KB}$ RAM. The Mbed is an easy-to-use tool, has a $\mathrm{C} / \mathrm{C}++$ programming environment, with an ARM-based compilation engine, suitable for fuzzy algorithm integration has a small size $(54 \times 26 \mathrm{~mm})$ and a low cost (USD 80).

The level measurement is generated with an SRF06 ultrasound sensor with a current output with a range of 4-20 mA, see Figure 3. This sensor is powered by a current loop and does not require other power; its measurement range is $2 \mathrm{~cm}$ to $510 \mathrm{~cm}$. The current output is four $\mathrm{mA}$ for a zero $\mathrm{cm}$ measurement and $20 \mathrm{~mA}$ at $510 \mathrm{~cm}$; the above gives a nominal current of $4 \mathrm{~mA}+31.37 \mathrm{uA} / \mathrm{cm}$. The SRF06 requires a loop drive voltage of $9 \mathrm{~V}$ to $24 \mathrm{~V}$, operates continuously automatically, and varies every 70-100 ms.

A single-phase KSI240 industrial relay with Triac or SCR at its output is used to activate the electric pump. It is usually used in industrial applications. This relay can be used for resistive, inductive, and capacitive loads. The control voltage is 4 to $32 \mathrm{Vdc}$ or 85 to $280 \mathrm{Vac}$, with a current output of $10 \mathrm{~A}, 25 \mathrm{~A}$, $40 \mathrm{~A}, 60 \mathrm{~A}, 80 \mathrm{~A}$ at 48-280 Vac, see Figure 4.

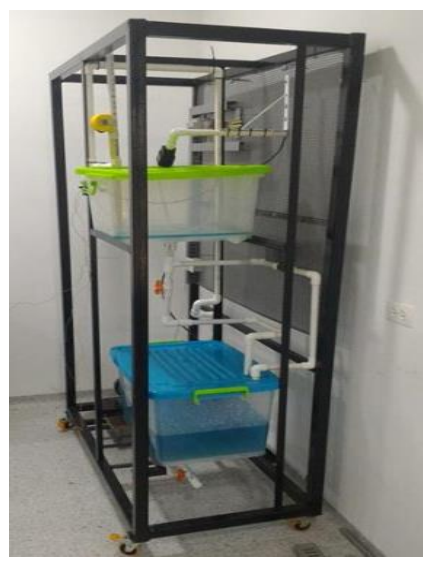

Figure 1. Coupled tanks station

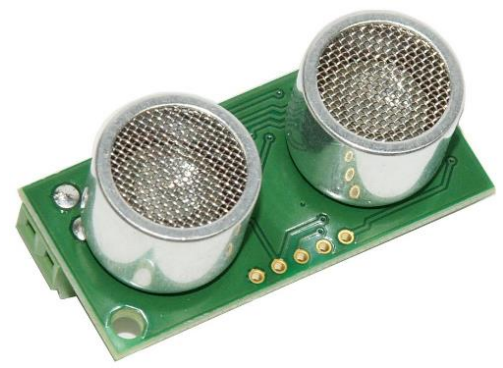

Figure 3. SRF 06 ultrasonic sensor

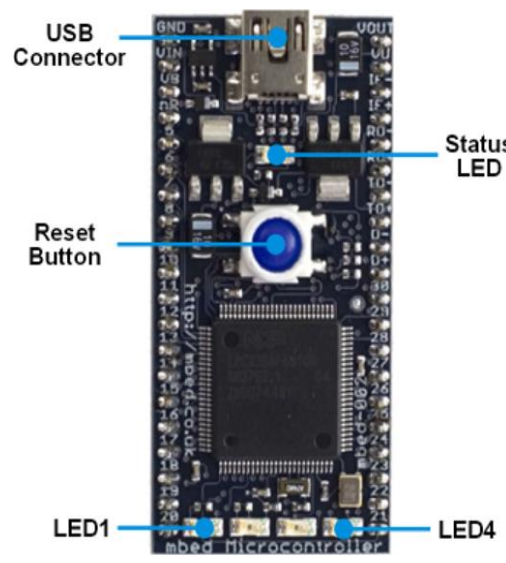

Figure 2. MBED NXP LPC1768 microcontroller

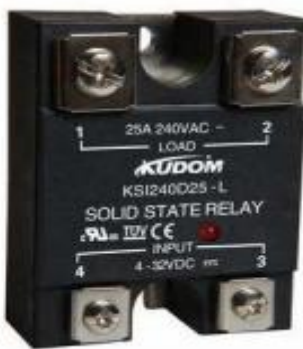

Figure 4. KSI240D25 relay 
The level controller design implies elaborating of a circuit for the synchronization signal from the microcontroller to the single-phase network or called a zero-crossing detector circuit. It is also necessary to generate a control signal for the electric pump motor, which corresponds to a signal pulse-width modulation (PWM) to turn the solid state relay (SSR) on or off. Figure 5 shows the circuit and the zero-crossing simulation signals. The yellow signal corresponds to the rectification stage's signal. The green one corresponds to the voltage on the $4 \mathrm{~N} 25$ optocoupler LED, the purple signal to the voltage on the optocoupler's resistance, which means the crossover detector's output. Likewise, the blue signal corresponds to conditioning to the input signal through a comparison and saturation stage. Its stage employs an LM324N and a $74 \mathrm{HC} 14 \mathrm{~N}$ that create the necessary pulse to enter the microcontroller.

Motor control requires the operation of the solid-state relay. The relay has a zero-crossing activation, regardless of the moment of the half-cycle in which the input signal is excited, waiting for the next zero-crossing to activate or deactivate the relay. Therefore, the minimum variation to be made for the PWM will be a half cycle as shown in Figure 6. This technique is known as zero cross turn-on and differs from the phase control technique called random turn on [5]. The Mbed microcontroller has a PWM output assigned to the output device and its respective variation in the whole operation of the SSR.
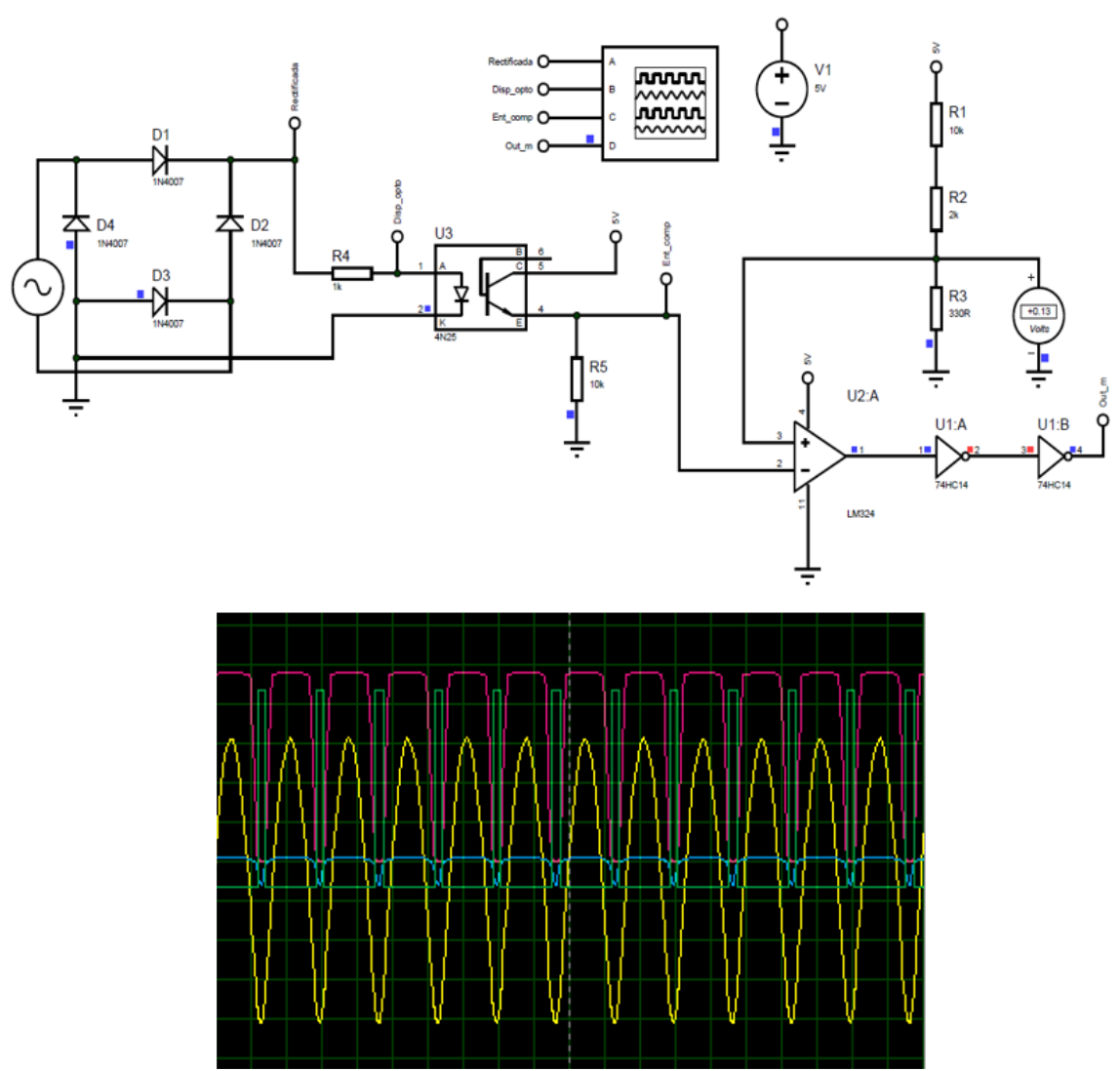

Figure 5. Circuit and the zero-crossing simulation signals
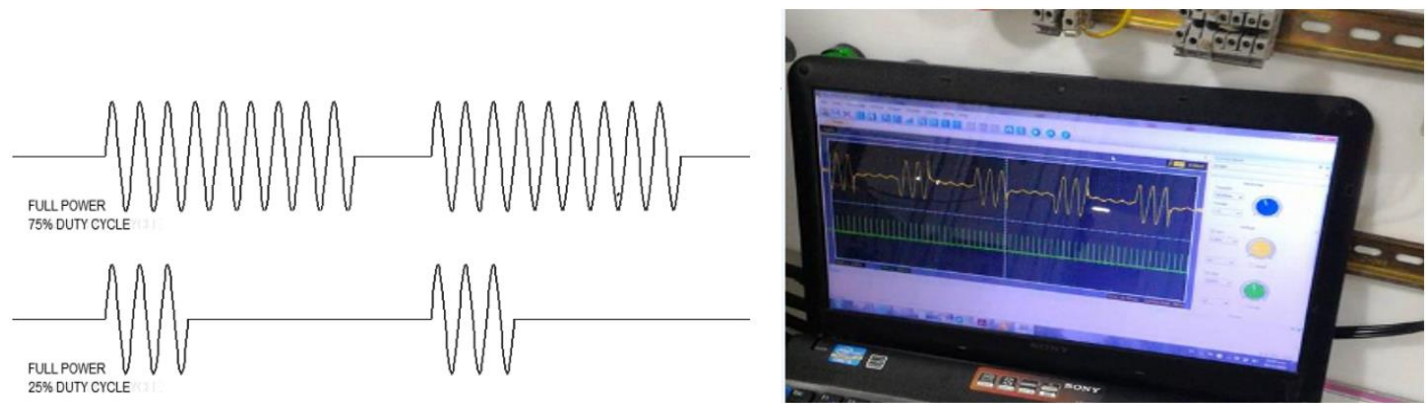

Figure 6. Expected and obtained output signal 


\subsection{Software}

A fuzzy Mamdani-type controller with proportional, integral, and derivative (PID) actions is embedded inside the microcontroller, with zero integral action and initially simulated in MATLAB as shown in Figure 7. The controller's two inputs correspond to the error and the derivative of the error and the output to the percentage of the required PWM signal's duty cycle. The pulse width-level relationship has been previously parameterized and identified; this allows identifying the PWM signal's duty-cycle and establishing a setpoint through the rule base. For example, a $67 \%$ duty cycle or controller output with a $20 \mathrm{~ms}$ period PWM signal allows setting a setpoint at $10 \mathrm{~cm}$.

Figure 8 allows to appreciate the input membership functions used in the controller design. The universe of discourse covers the range from 0 to 250 centimeters for the input error, and a maximum variation percentage of 10 percent was defined for the rate of change of the derivative input. Linguistic labels of medium-low (ml), low (l), zero (z), high (h) and medium-high ( $\mathrm{mh}$ ) were used [26]. Figure 9 shows the output membership functions for the duty cycle of the PWM signal, with linguistic labels of super-low (SL), low low (LL), low (L), medium (M), high (H), and high (HH) and super high (SH).

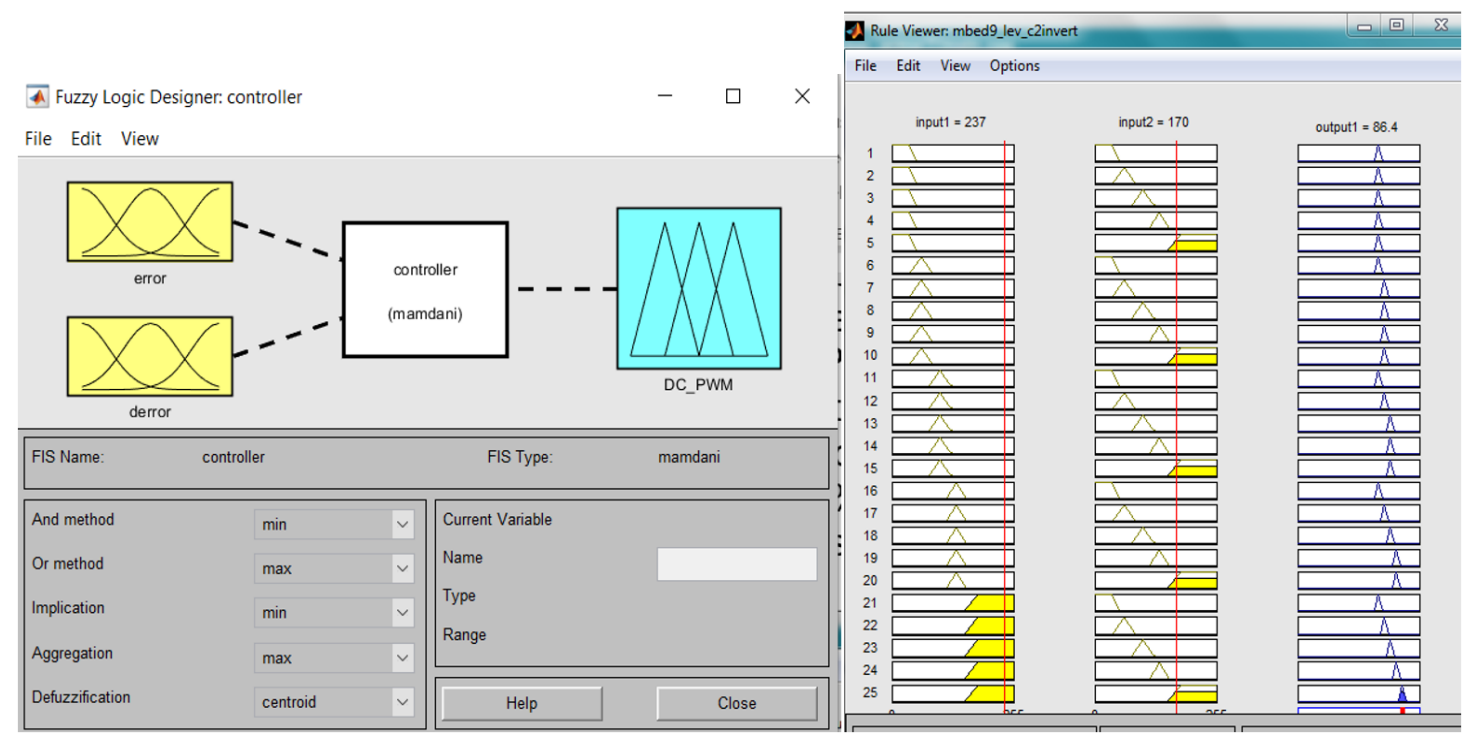

Figure 7. Mamdani fuzzy block and the activation of each of the rules
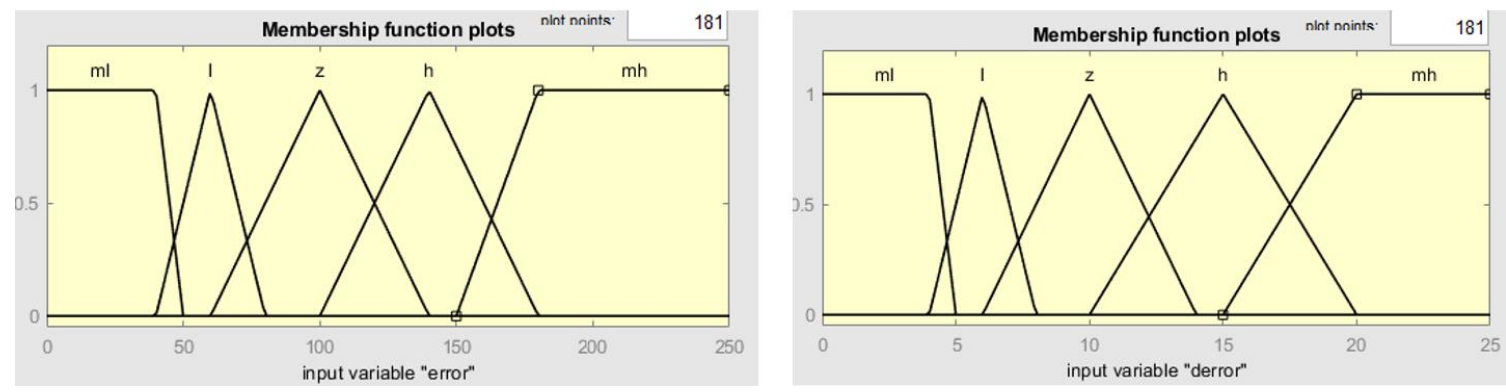

Figure 8. Defining input sets

Simulation of the controller allows obtaining the control surface illustrated in Figure 10, where the gradual increase in the output of the duty cycle is seen, smoothed, and continuous, which determines a stable control action for the system. Once the operation of the controller is validated, it is transcribed into $\mathrm{C}$ language for the microcontroller. An interface was created in LabView for the visualization of the plant level, taking advantage of the peripherals of the serial communication microcontroller, where the level data is sent and allows the user to visualize, through a graphic representation of the tank in real-time, the measurement value and the drive of the electric pump as illustrated in Figure 11. 


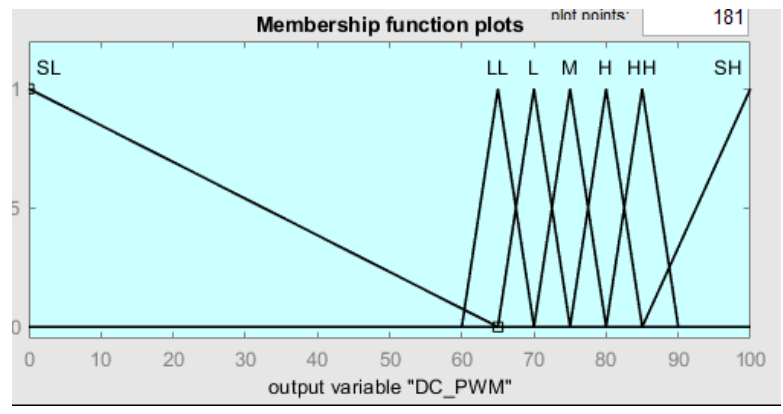

Figure 9. Effects of selecting different switching under dynamic condition

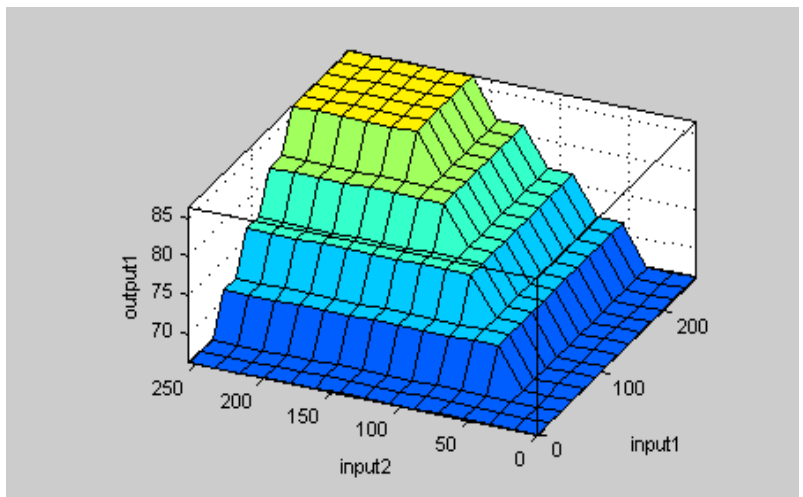

Figure 10. Rule base generated surface, output, and inputs

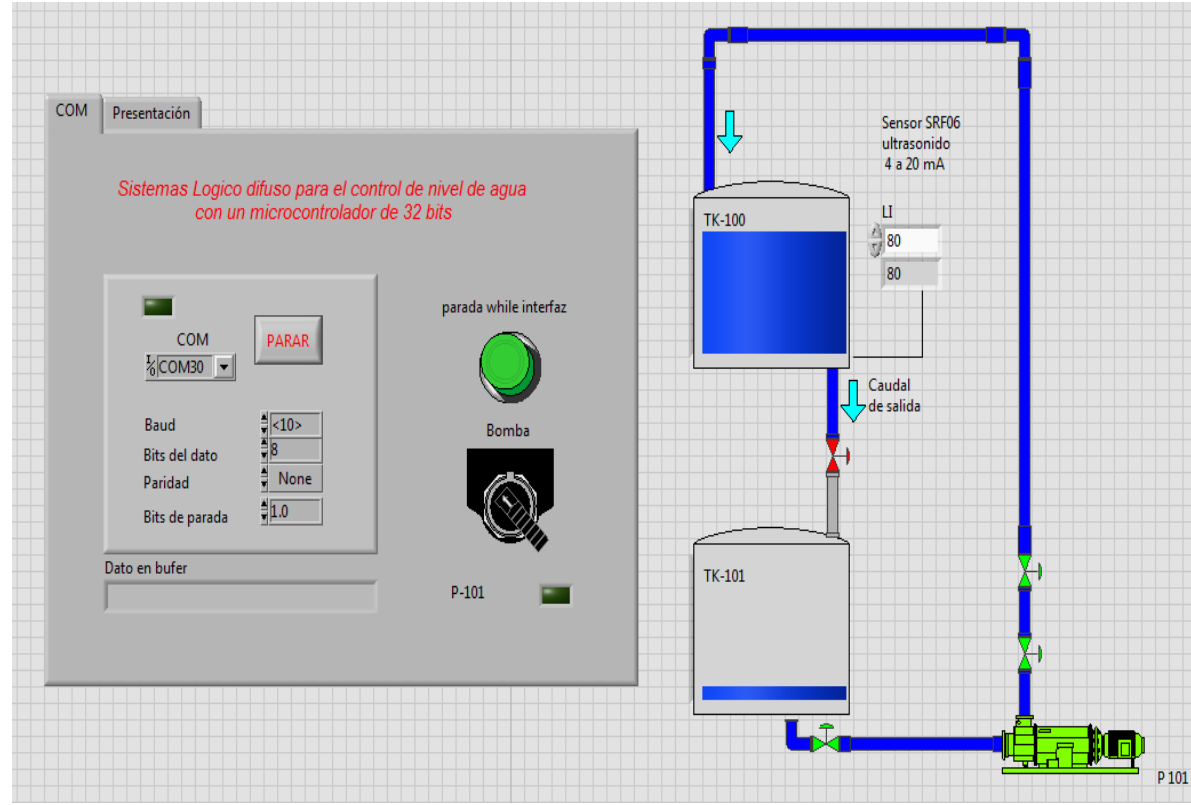

Figure 11. Visualization software

\section{RESULTS AND DISCUSSION}

Once the designs and simulations are done, the controller circuits are implemented. Figure 12 shows the power circuit to power the microcontroller, an LCD as a local display interface, a keyboard, and the level sensor. Figure 13 shows the controller circuit. It contains the Mbed microcontroller that, using a matrix keyboard and the LCD, performs the reading and entry of parameters for the level control system's proper operation. 


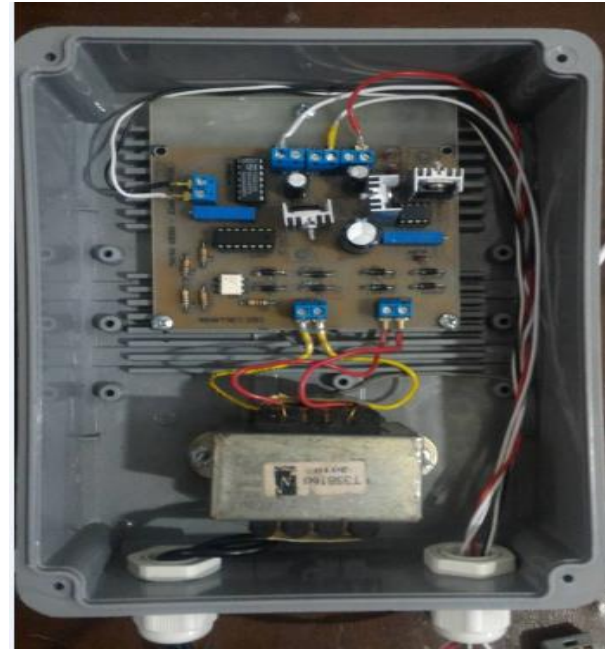

Figure 12. Sensor supply circuit and zero crossing

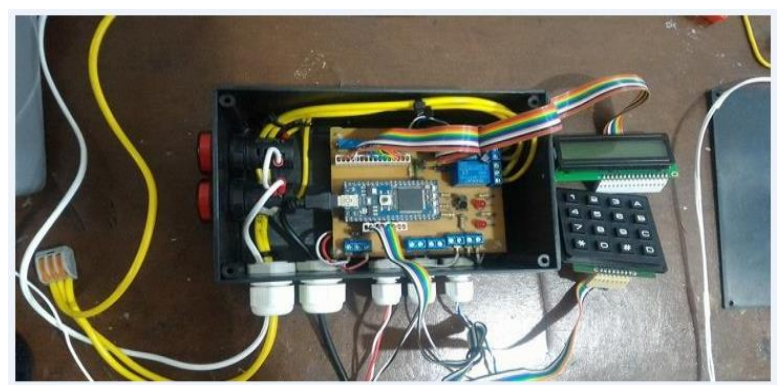

Figure 13. Control circuit. Mbed, 4x4 keyboard and 16x2 LCD

The SRF06 sensor used has a protective casing as shown in Figure 14, making it robust to the environment, preventing it from being exposed to water and dust that can occur in a harsh work environment. For the development of the prototype, a restored workbench carcass was used and later modified to use water storage tanks. As a result, a stable, robust, and broad structure for level control is obtained. Table 1 shows the performance of the controller when adjusting different set-points. The steady-state error is evidenced, which is low, and in turn the response time obtained to reach the system establishment is evidenced. In no case was there evidence of maximum overshoot exceedance of $8 \%$.

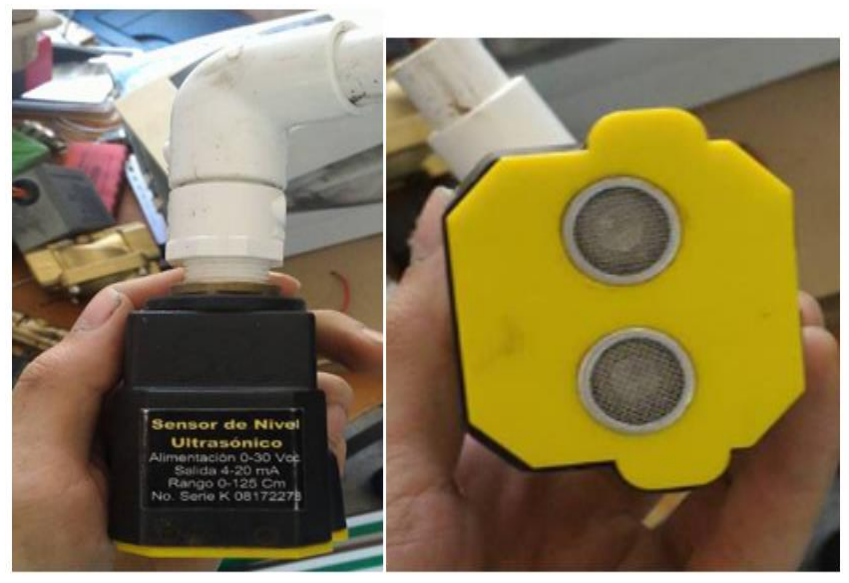

Figure 14. SRF06 ultrasonic sensor installation (4-20 mA) 
Table 1. The performance of fuzzy controller

\begin{tabular}{ccc}
\hline Setpoint & Ess & $\operatorname{Tr}$ \\
\hline $180 \mathrm{~cm}$ & $1.8 \%$ & $223 \mathrm{seg}$ \\
$200 \mathrm{~cm}$ & $1.92 \%$ & $268 \mathrm{seg}$ \\
$220 \mathrm{~cm}$ & $2.03 \%$ & $311 \mathrm{seg}$ \\
\hline
\end{tabular}

\section{CONCLUSION}

It was possible to obtain a prototype with independent power and remote supervision through LabView, which efficiently controls the desired water level of coupled tanks. The embedded controller results in a portable system that can be adapted to other control schemes with only an additional tuning process. The computational model of the fuzzy controller enables establishing the design conditions based on the two types of input sets, the error, and its derivative. Also, it allows generating the rule base for the control action and variation of specific input setpoints through the knowledge-based analysis of the system. Lastly, the stability and the system response analysis showed a suitable performance under the expert criteria, based on the simplicity of the final design and the desired control parameters.

\section{ACKNOWLEDGEMENTS}

The authors are grateful to Universidad de los Llanos and Universidad Militar Nueva Granada for the funding of this project.

\section{REFERENCES}

[1] A. N. Abdullah and M. H. Ali, "Direct torque control of IM using PID controller," International Journal of Electrical and Computer Engineering (IJECE), vol. 10, no. 1, pp. 617-625, 2020, doi: 10.11591/ijece.v10i1.pp617-625.

[2] M. O. Rachedi, M. L. Saidi, and F. Arbaoui, "MPPT control design for variable speed wind turbine," International Journal of Electrical and Computer Engineering (IJECE), vol. 10, no. 5, pp. 4604-4614, 2020, doi: 10.11591/ijece.v10i5.pp4604-4614.

[3] N. Hou and Y. Li, "A direct current control scheme with compensation operation and circuit-parameter estimation for full-bridge DC-DC converter," IEEE Transactions on Power Electronics, vol. 36, no. 1, pp. 1130-1142, 2021, doi: 10.1109/TPEL.2020.3002737.

[4] Z. Muhammad, M. A. A. M. Hafez, N. A. M. Leh, Z. M. Yusoff, and S. A. Hamid, "Smart agriculture using internet of things with raspberry Pi," 2020 10th IEEE International Conference on Control System, Computing and Engineering (ICCSCE), Penang, Malaysia, 2020, pp. 85-90, doi: 10.1109/ICCSCE50387.2020.9204927.

[5] S. A.-Cabrera, G. P.-Valenzuela, J. P.-Jara, and A. R.-Moreno, "Decentralized control of a water tank using MBPC control," 2020 IEEE XXVII International Conference on Electronics, Electrical Engineering and Computing (INTERCON), Lima, Peru, 2020, pp. 1-4, doi: 10.1109/INTERCON50315.2020.9220264.

[6] A. T. Hoang, T. V. Nguyen, and B. T. Nguyen, "The novel design of feed-water control system for thermal power plant using super-critical start-up motor-boiler feed-water pump," 2020 IEEE PES/IAS PowerAfrica, Nairobi, Kenya, 2020, pp. 1-5, doi: 10.1109/PowerAfrica49420.2020.9219903.

[7] K. O. Akpinar and I. Ozcelik, "Anomaly detection on etherCAT based water level control automation," 2020 5th International Conference on Computer Science and Engineering (UBMK), Diyarbakır, Turkey, 2020, pp. 79-82, doi: 10.1109/UBMK50275.2020.9219391.

[8] R. T.-Cabezas, "A hybrid fuzzy modeling method to improve the strategic scenarios design: Integrating artificial intelligence algorithms and the field of futures studies methods," 2021 16th Iberian Conference on Information Systems and Technologies (CISTI), 2021, pp. 1-6, doi: 10.23919/CISTI52073.2021.9476261.

[9] F. Röben and A. C. Meissner, "Market response for real-time energy balancing with fuzzy logic," 2020 17th International Conference on the European Energy Market (EEM), Stockholm, Sweden, 2020, pp. 1-6, doi: 10.1109/EEM49802.2020.9221945.

[10] X. Fan, P. Bai, H. Li, X. Deng, and M. Lv, "Adaptive fuzzy finite-time tracking control of uncertain non-affine multi-agent systems with input quantization," IEEE Access, vol. 8, pp. 187623-187633, 2020, doi: 10.1109/ACCESS.2020.3030282.

[11] Y. Wu, H. Xue, Y. Tian, and H. Liang, "Adaptive fuzzy finite-time bipartite containment control for stochastic multi-agent systems," 2020 International Conference on System Science and Engineering (ICSSE), Kagawa, Japan, 2020, pp. 1-6, doi: 10.1109/ICSSE50014.2020.9219267.

[12] Y. Fan and Y. Li, "Adaptive fuzzy finite-time fault-tolerant control for uncertain non-strict feedback nonlinear systems," 2020 International Conference on System Science and Engineering (ICSSE), Kagawa, Japan, 2020, pp. 1-6, doi: 10.1109/ICSSE50014.2020.9219326.

[13] H. V.-Hinostroza and A. R.-Moreno, "Real-time model reference adaptive fuzzy control of a 3DOF robot arm," 2020 IEEE XXVII International Conference on Electronics, Electrical Engineering and Computing (INTERCON), Lima, Peru, 2020, pp. 1-4, doi: 10.1109/INTERCON50315.2020.9220247.

[14] A. N. Mohammed and G. A. -R. Ghoneim, "Fuzzy-PID speed controller model-based indirect field oriented control for induction motor," 2020 International Conference on Computer, Control, Electrical, and Electronics Engineering (ICCCEEE), 2021, pp. 1-6, doi: 10.1109/ICCCEEE49695.2021.9429623.

[15] A. Kasbi and A. Rahali, "Performance optimization of doubly-fed induction generator (DFIG) equipped variable-speed wind energy turbines by using three-level converter with adaptive fuzzy PI control system," Materials Today: Proceedings, 2021, doi: 10.1016/j.matpr.2021.05.406.

[16] Q. Bu et al., "The effect of fuzzy PID temperature control on thermal behavior analysis and kinetics study of biomass microwave pyrolysis," Journal of Analytical and Applied Pyrolysis, vol. 158, 2021, Art. no. 105176, doi: 10.1016/j.jaap.2021.105176.

[17] R. Alika, E. M. Mellouli, and E. H. Tissir, "Adaptive higher-order sliding mode control based fuzzy logic T-S for lateral dynamics of autonomous vehicles," 2021 12th International Conference on Information and Communication Systems (ICICS), 2021, pp. 358-363, doi: 10.1109/ICICS52457.2021.9464623. 
[18] N. D. Lopes, K. Guelton, L. Arcese, and V. J. S. Leite, "Local sampled-data controller design for T-S fuzzy systems with saturated actuators," IEEE Control Systems Letters, vol. 5, no. 4, pp. 1169-1174, 2021, doi: 10.1109/LCSYS.2020.3019215.

[19] R. Jimenez, C. Caceres, O. Avilés, and C. Grodillo, "Multi-tank fuzzy level controller system using system," 2012 IEEE Ninth Electronics, Robotics and Automotive Mechanics Conference, Cuernavaca, 2012, pp. 320-324, doi: 10.1109/CERMA.2012.77.

[20] J. S. Furter and P. C. Hauser, "Interactive control of purpose built analytical instruments with forth on microcontrollers-a tutorial," Analytica Chimica Acta, vol. 1058, 2019, pp. 18-28, doi: 10.1016/j.aca.2018.10.071.

[21] K. L. Cézar et al., "Development of a novel flow control system with Arduino microcontroller embedded in double effect absorption chillers using the $\mathrm{LiBr} / \mathrm{H} 2 \mathrm{O}$ pair," International Journal of Refrigeration, vol. 111, pp. 124-135, 2020, doi: 10.1016/j.ijrefrig.2019.11.014.

[22] R. R. Rout, S. Vemireddy, S. K. Raul, and D. V. L. N. Somayajulu, "Fuzzy logic-based emergency vehicle routing: An IoT system development for smart city applications," Computers \& Electrical Engineering, vol. 88, 2020, Art. no. 106839, doi: 10.1016/j.compeleceng.2020.106839.

[23] A. S. Fonseca Sobrinho and F. G. Junior, "Type-1 fuzzy logic algorithm for low cost embedded systems," Computers \& Electrical Engineering, vol. 88, 2020, Art. no. 106861, doi: 10.1016/j.compeleceng.2020.106861.

[24] J. M.-Gil and J. M. Chaves-Gonzalez, "Automatic design of semantic similarity controllers based on fuzzy logics," Expert Systems with Applications, vol. 131, pp. 45-59, 2019, doi: 10.1016/j.eswa.2019.04.046.

[25] R. Alimuin, E. Calilung, and E. Dadios, "Implementation of machine vision as an input to a fuzzy water level control system," 2017 IEEE 9th International Conference on Humanoid, Nanotechnology, Information Technology, Communication and Control, Environment and Management (HNICEM), 2017, pp. 1-6, doi: 10.1109/HNICEM.2017.8269484.

[26] R. Babuska, "Fuzzy and neural control," DISC Course Lecture Notes (October 2001), Faculty of Information Technology and Systems. Delft University of Technology, Delft, the Netherlands, 2001.

\section{BIOGRAPHIES OF AUTHORS}

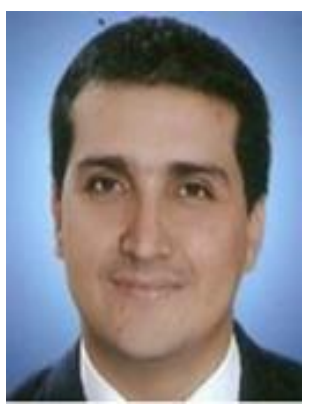

Javier Eduardo Martinez Baquero (D) SC S P is an Electronic Engineer graduated from Universidad de los Llanos in 2002. Postgraduate in Electronic Instrumentation from Universidad Santo Tomas in 2004, Postgraduate in Industrial Instrumentation and Control from Universidad de los Llanos in 2020 and M.Sc. Educative Technology in Universidad Autonoma de Bucaramanga in 2013. He can be contacted at email:jmartinez@unillanos.edu.co.

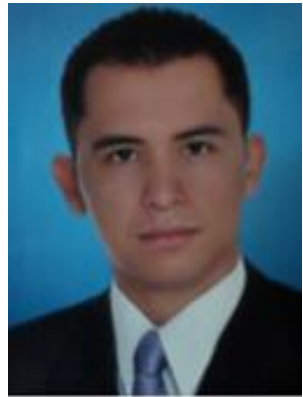

Jairo David Cuero Ortega (DD SI SC P is an Electronic Engineer graduated from Universidad de los Llanos (Unillanos) in 2012. He also holds a postgraduate degree in Industrial Instrumentation and Control from the same university. Currently, he is studying Master of Engineering-Industrial automation at Universidad Nacional de Colombia. He works as a professor at Unillanos, and his research scopes are Instrumentation, Control and Robotics. He can be contacted at email: jairo_cuero@unillanos.edu.co.

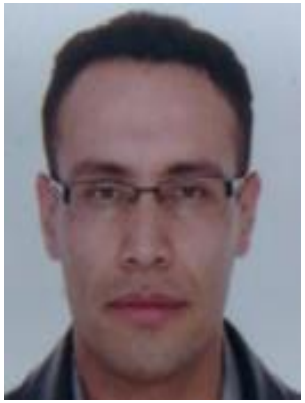

Robinson Jiménez-Moreno (D) SC SC is an Electronic Engineer graduated from Universidad Francisco José de Caldas in 2002. He received a M.Sc. in Engineering from Universidad Nacional de Colombia in 2012 and Ph.D. in Engineering at Universidad Francisco José de Caldas in 2018. Currently, he is working as an assistant professor of Universidad Militar Nueva Granada and his research focuses on convolutional neural networks for object recognition and image processing for robotic applications such as human-machine interaction. He can be contacted at email: robinson.jimenez@unimilitar.edu.co. 